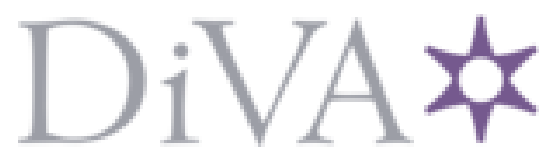

http://www.diva-portal.org

\title{
Postprint
}

This is the accepted version of a paper presented at 2016 IEEE Power and Energy Society General Meeting (PESGM).

Citation for the original published paper:

Bogodorova, T., Vanfretti, L., Turitsyn, K. (2016)

Voltage Control-Based Ancillary Service using Thermostatically Controlled Loads.

In: 2016 IEEE Power and Energy Society General Meeting (PESGM) IEEE

http://dx.doi.org/10.1109/PESGM.2016.7741640

N.B. When citing this work, cite the original published paper.

Permanent link to this version:

http://urn.kb.se/resolve?urn=urn:nbn:se:kth:diva-198088 


\section{Voltage Control-Based Ancillary Service using Thermostatically Controlled Loads}

\author{
Tetiana Bogodorova \\ KTH Royal Institute of Technology \\ Stockholm, Sweden \\ Email: tetianab@kth.se
}

\author{
Luigi Vanfretti \\ KTH Royal Institute of Technology \\ Stockholm, Sweden \\ Statnett SF \\ Oslo, Norway \\ Email: luigi.vanfretti@ee.kth.se
}

\author{
Konstantin Turitsyn \\ Department of Mechanical Engineering \\ Massachusetts Institute of Technology \\ Cambridge, MA 02139-4307 \\ Email: turitsyn@mit.edu
}

\begin{abstract}
This paper discusses the possibility of providing voltage control-based ancillary services using thermostatically controlled loads (TCLs). The idea is to change the voltage at the point of common coupling in order to control power consumption of the TCLs through time. This allows to utilize the most common type of loads in the grid to provide ancillary services. These services differ from usual demand response strategies that operate on short to medium time scales. In this paper the authors propose a controller that regulate the power consumption by minimizing the power consumption error signal and by taking physical restrictions into account. Voltage restrictions arise due to the small window of voltage variability about the nominal value of the controlled voltage. In addition, capacitor bank switching interactions with motor and thermostatically controlled loads is discussed.
\end{abstract}

Index Terms - Thermostatically controlled loads, voltage control, load modeling, ancillary services, HVAC.

\section{INTRODUCTION}

One of typical ancillary service, which is considered in this article, is energy imbalance management or load following ${ }^{1}$. The distinctive characteristic of this service is that regulation relies not only on the Real-Time Energy (PJM) or Regulating Power Market (Nordic), but also on the network capacity (loads) present in the system to be able to maintain balance in the grid (operating in a small energy range). The technical requirements for such type of ancillary services demand continuous metering (synchrophasors (PMU), supervisory control and data acquisition (SCADA) or interval data recorder (IDR) measurement data). In addition, some transmission system operators (TSOs) require to have a minimum size of load capacity reserves to meet regulation requirements, especially those who consider imbalance energy services as real-time response services (stringent response requirements). The latter greatly depend on time frame response rules and network characteristics (size, reliability, communication and control

T. Bogodorova and L. Vanfretti are supported by the EU funded FP7 iTesla project.

L. Vanfretti is supported by Statnett SF, Oslo, Norway.

K. Turitsyn is with Department of Mechanical Engineering, Massachusetts Institute of Technology, Cambridge, USA.

${ }^{1}$ Energy imbalance management or load following is defined in [1], and it is used to ensure balance between demand and supply in a time window of ten minutes to hours (see pp.6 and Table 3 in [1]) constraints, etc.).

Ancillary services differ from usual demand response strategies that operate on short to medium time scales. ${ }^{2}$ The most appropriate type of loads to provide ancillary services are thermostatically controlled loads (TCLs). The potential of TCLs has been broadly discussed in the scientific [3], [4] and industrial circles [5]; while controllability of electrical loads is a current research topic [6]. Differently from Conservation Voltage Reduction (CVR) [7] which aims to reduce energy consumption resulting from a reduction of feeder voltage, the goal of this paper is to provide ancillary services controlling voltage and active power. In this context, an additional controller into the load tap changing control loop is proposed in this paper.

The goal of this article is to investigate and assess the ability to maintain network balance when controlling the voltage signal at the point of common coupling (PCC) where a load tap changer (LTC) is placed. Assuming a large amount of TCLs (which is the most common type of load in distribution networks), it is possible to aggregate a sufficiently large load power consumption response change (smoothing) over time to maintain ancillary service requirements. This change can be achieved by varying the voltage in a small (3\%-5\%) range, while respecting constrains of other control devices.

The remainder of this article is structured as follows. Section II discusses the main idea of the article. Section III offers a description of different load model types. Section IV shows the regulator design aimed to control the active power consumption of TCL loads by using voltage as a natural control signal. Section V provides more insight into the particularities of the nonlinear model of the load and its control. Section VI sums up results, draws conclusions, and discusses future work.

\footnotetext{
${ }^{2}$ As described in [2] demand response focuses on emergency load relief and peak load management or price responsive demand. For ancillary services technical requirements are more challenging in terms of speed and accuracy; the energy component is small relative to capacity. Furthermore, ancillary services are needed year-round and not just during peak hours. Ancillary services involve small amounts of energy, but their real value is in the capacity held in reserve and the technical capability to respond reliably and quickly to maintain balance (for more details see [2]).
} 


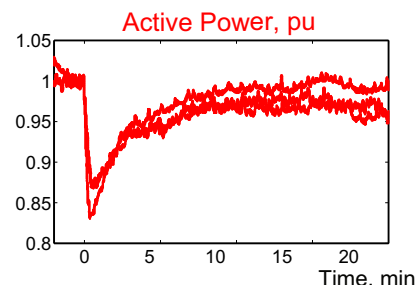

Fig. 1: Active power recordings [14]

\section{Controlling the Voltage Signal}

A number of works on aggregated load modeling published in recent decades [8], [9], [10], [11] studied the mathematical representation of loads through a fractional exponential dependency of power consumption with respect to voltage (omitting reactive power representation) and its influence on power system stability [12]:

$$
P_{1}=P_{0}\left(\frac{V_{1}}{V_{0}}\right)^{\alpha}
$$

where the exponent $\alpha$ determines the sensitivity of the load power to the voltage with respect to voltage variations around the reference operating point $\left(P_{0}, V_{0}\right)$, that is:

$$
\alpha=\frac{d P / P_{0}}{d V / V_{0}}
$$

According to [13] a deviation of the voltage in $1 \%$ causes change in active power consumption around $1 \%$ [2]. This helps fulfilling the requirements for responsive reserve services. Similar observations have been made in [14], and state that voltage disturbances lead to power recovery to a new steady state. Figure 1 illustrates this phenomenon, as observed from field measurements. This nonlinear response to the voltage level offers an opportunity for providing ancillary services by modulating the voltage level on distribution feeders. The main advantage of this approach is its relatively low cost in comparison to more technologically complicated solutions involving smart thermostats and an additional layer of communication and control infrastructure. Indeed, regulation of the voltage at the load side can be achieved via a number of existing technologies, like load tap changers (LTCs), capacitor banks, and automatic voltage regulation on generators [15]. At the same time, sensing and control can be accomplished via existing SCADA and energy management/ outage management systems (EMS/OMS) that are already used for voltage regulation, or using phasor measurement units that are currently being deployed by many utilities.

There are several drawbacks of the proposed approach. The first one relates to the relatively low range of voltage levels that are acceptable for normal power system operation. Typically, this range is confined to $10 \%$ of the nominal voltage. As the systems tend to operate close to the voltage limits, this implies that, in most of the system, voltage variations will need be confined to $2-5 \%$. Hence, the response in the voltage will be similarly small. However, it may be enough to provide ancillary services that require only about $1 \%$ of flexibility of typical power consumption levels [2].
The second drawback wear costs incurred by system owners by frequent switching of tap changers and capacitor banks. Every switching event will add to equipment wear and reduction in its lifetime. Although this may be a serious obstacle for aging systems, it will become less of an issue as more power electronic devices (static var compensator (SVC), static synchronous compensators (STATCOMs), and even simple inverters) are introduced in the system. Such devices offer a unique opportunity for distributed voltage regulation [16] and may be naturally exploited through the schemes discussed in this article.

The third issue is the lack of load response predictability. The load composition, as well as environmental conditions such as temperature, are continuously changing. Thus, the system needs to rely on control loops that adapt to constantly changing conditions. Solutions to this problem are discussed in the following sections.

Finally, another important problem is the long-term recovery of the load level. Typically, individual devices are operated via a sequence of control loops that provide feedback on different time-scales. Hence, the recovery to the original power consumption level may occur on time-scales much longer than the original transient. The most important example is the recovery of the power consumption of an ensemble of TCLs (e.g. heating, ventilating, and air conditioning (HVACs)) that occurs on time-scales comparable to the typical dutycycle of the devices. This recovery limits the bandwidth of the disturbances that the voltage regulated loads can respond to and requires more sophisticated models to be used in analysis and synthesis of control loops. Controllers should thus consider both the short- and long-term response of the system to voltage variations have to be introduced. These types of control and analysis models are introduced in the following sections.

\section{LOAD MODELING}

This section provides the model of a single TCL and induction motor model characteristics which can contribute to voltage control-based ancillary service .

\section{A. Induction motor model}

Most of the TCLs are operated by induction motors that are used to transfer the mediums involved in heat exchange, e.g. air in air conditioners, water in district heating systems, and organic working fluids in refrigerators. The electrical torque of the motor depends on the motor's terminal voltage and slip. In steady state the electrical torque will be balanced by the mechanical torque, that for most of the TCLs has a quadratic dependence on the speed. Hence, the drop in the voltage will induce a small drop in the velocity, and consequently, drop in power (Figure 2). The exact sensitivity depends on the speed-torque profile of the motor, mainly through the impedances of the rotor and stator. More resistive loads will be more sensitive to voltage variations, whereas lightly loaded motors with high efficiency will be less. The following section proposes a generic power dependency on the voltage that 


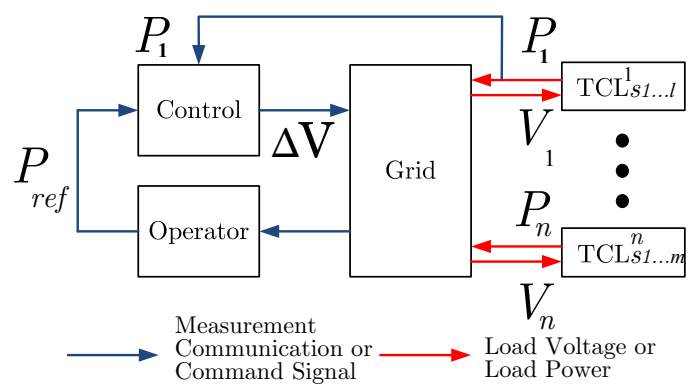

Fig. 3: Block diagram of the control infrastructure and communication between the controller and the grid

will need to be adjusted to specific systems in more detailed analysis.

\section{B. Thermostatically Controlled Loads (TCLs) model}

The TCLs are described using a model that is common in the literature [3], [17]. Each individual TCL model is represented using the model:

$$
\frac{\partial \theta}{\partial t}=\frac{1}{\tau}\left(-\theta_{a}+\theta+P R\right)
$$

where $\theta$ - temperature $\left[{ }^{\circ} \mathrm{C}\right] ; P$ - power consumption [kW], $V$ - voltage $[\mathrm{V}], C$ - thermal capacitance $\left[\mathrm{kWh} /{ }^{\circ} \mathrm{C}\right], R$ thermal resistance $\left[{ }^{\circ} \mathrm{C} / \mathrm{kW}\right], R_{r}$ - rated resistance, $\theta_{a}$ - ambient temperature $\left[{ }^{\circ} \mathrm{C}\right], \tau$ equals $C * R$, and $P$ equals $u(t) *$ $V^{2}(t) / R_{r}$. Subject to the switching condition:

$$
u(t+1)= \begin{cases}u(t) & \text { if } \theta_{\min } \leq \theta \leq \theta_{\max } \\ 1-u(t) & \text { otherwise }\end{cases}
$$

where $u(t)=1$ if the TCL is on, $u(t)=0$ if the TCL is off, and $u(t)$ otherwise. The change in power consumption is quadratically proportional to the voltage change.

\section{Model Of Controller}

A simplified communication infrastructure between the controller located at the distribution substation level and the grid is shown in Fig. 3. The control block changes the voltage comparing an operator's set point (power consumption demand) and power measurements on the loads. It is assumed that the operator sets power references that respect load capacity limits to provide the required consumption (i.e. hidden reserves of the loads). Voltage regulation is usually performed by tap changer on distribution transformers. The load tap changer controls the voltage of the power distribution side by changing the transformer tap ratio [9]. For simplicity, a continuous LTC model was chosen for simulation:

$$
\begin{aligned}
& T_{c} \frac{d r}{d t}=V_{2}-V_{2}^{0}, \\
& r_{\min } \leq r \leq r_{\max }
\end{aligned}
$$

where $T_{c}$ - a control time constant, $r$ - a continuously changing tap, $V_{2}$ is the distribution side voltage, and $V_{2}^{0}$ - its reference value.

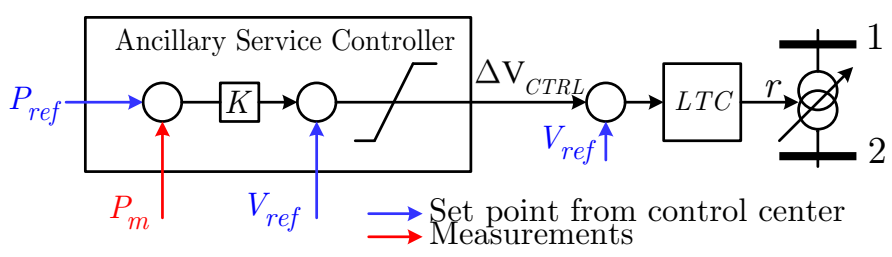

Fig. 4: Controller and LTC in cascade

As a proof of concept, a proportional control together with the LTC are used to control the voltage change. ${ }^{3}$ This allows to use tap changing devices to provide the ancillary service. In addition, it is a very cheap solution as compared to installing multiple sensors and controllers at the customer side. The controller was modeled using the Modelica language and is shown in Figure 4. The internal structure of the controller can be described as follows:

$$
\begin{aligned}
V_{\text {out }}-V_{\text {ref }} & =k\left(P_{\text {ref }}-P\right), \\
V_{\text {min }} & \leq V_{\text {out }} \leq V_{\text {max }}
\end{aligned}
$$

The proportional coefficient can be chosen according to regulation quality indicators and can be tuned later on.

\section{Simulation Cases and Result Analysis}

In this section several numerical experiments and analyses are presented to prove the feasibility to provide the ancillary service discussed in Section II by using the voltage as a natural regulation signal.

Each experiment $(1(a, b), 2(a, b))$ corresponds to a different network configurations and type of disturbance. The configurations are of 3 types: 1) with or without LTC - to observe LTC action under different type of disturbances; 2) with or without control - to observe the controller performance; 3) two types of active power references as controller input serving the main goal. The disturbances were chosen of the 3 types: no fault, small perturbation and load shedding. Each case has to serve the goal to get demanded power consumption change by varying the voltage of the power distribution side. All the experiments and its results are summarized in Table I. In order to consider stochastic behavior of TCLs, the distribution of each signal was estimated, mean and variance where summarized.

\section{A. Case 1: Change in power consumption for a TCL load $(n=22)$}

The power system models that are used for different case studies include homogeneous and heterogeneous groups of TCLs. Each TCL has the same thermal resistance $R=$ $200^{\circ} \mathrm{C} / \mathrm{kW}$, power consumption $P=0.14$ p.u, $\theta_{a}=32^{\circ} \mathrm{C}$, switching temperature range of $[19.75 . .20 .25]^{\circ} \mathrm{C}$. Heterogeneous TCLs $(\mathrm{n}=10)$ differ in parameter $C$ in the range $[2.0 . .4 .0571]^{\circ} \mathrm{C}, 2$ of TCLs ( $\left.\mathrm{n}=5\right)$ of $[2.0 . .2 .9143], 2$ of TCLs

\footnotetext{
${ }^{3}$ Observe that conventional LTC controls are untouched and the additional voltage control signal $V_{\text {out }}$ is introduced to the voltage error summing junction of the LTC as $\Delta V_{L T C}=\left(V_{L T C r e f}-V_{m}\right)+V_{\text {out }}$, which the LTC uses for its conventional regulation function
} 


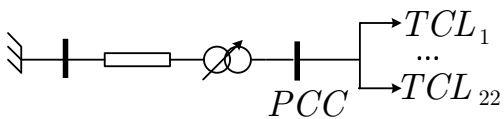

Fig. 5: Model of Experiment 1 (a - inactive LTC, b - active LTC) with TCL loads $(n=22)$

$C=2.9143$. The load tap changer (LTC) model used is the same as in [18] working in the range [0.97..1.03] having 32 positions (Fig. 5).

The simulation time of the experiments is 1000 sec. For the sake of statistical adequacy time intervals, before and after the disturbances are applied, are the same $(t=500 \mathrm{sec})$. A small perturbation is created by disconnecting a single TCL. It corresponds to a $4.5 \%$ load shed of the total load. A large perturbation is equivalent to the disconnection of 5 TCLs or $23 \%$ shedding. Considering the slow dynamics of TCLs, the $3-$ phase fault on the bus is applied for 1 second at $t=100 \mathrm{sec}$ should not drastically change the power and voltage profile much unless the fault was not cleared, in such case the TCLs were switched off.

In experiment 1a (Table I) a distribution feeder with TCL loads and no active LTC (Fig. 5) is simulated in order to demonstrate the ability of active power consumption to shift via voltage regulation. The impedance of lines was neglected.

In the experiment $1 \mathrm{~b}$ (Table I) the same model as for $1 \mathrm{a}$ was exploited, but LTC is active in this time. The simulation starts from $50 \%$ of TCLs state being switched on or off.

The simulation results from experiment 1a show an active power consumption drop and a small increase in the mean of the voltage. The variance of the voltage and power signals has considerably decreased in the case of large load shedding. This can be explained by the fact that the disconnected loads were homogeneous in their parameters with respect to those left switched on. The logical conclusion that can be drawn is that heterogeneity results in a smoother profile of the voltage and power signals.

The results of experiment $1 \mathrm{~b}$ include the dynamics of LTC responding to the disturbances. In the case of most severe load disconnection, the voltage has increased and the upper voltage limit has been activated at the LTC. Consequently, due to it's action, the voltage has decreased. Conversely, because the $5 \%$ load shed is too small to activate the LTC, the voltage mean just increases after the disturbance is applied.

\section{B. Case 2: Control of power consumption of a TCL loads $(n=22)$}

This experiment (Fig. 4$)^{4}$ aims to control the voltage on the higher side of the transformer by using the regulator proposed in Section IV. Communication delays in the control feedback signal transmission and processing are neglected. To analyze the controller's performance, the power reference has been set to a constant value (experiment 2a) or as a step change (experiment $2 b$ ). Simulation results show that the power consumption is shifted due to the change of duty cycle

\footnotetext{
${ }^{4}$ The model in Fig. 4 is extended by adding an infinite bus at bus 1 and 22 independent TCLs defined by (3) with parameters defined in Section V-A.
}
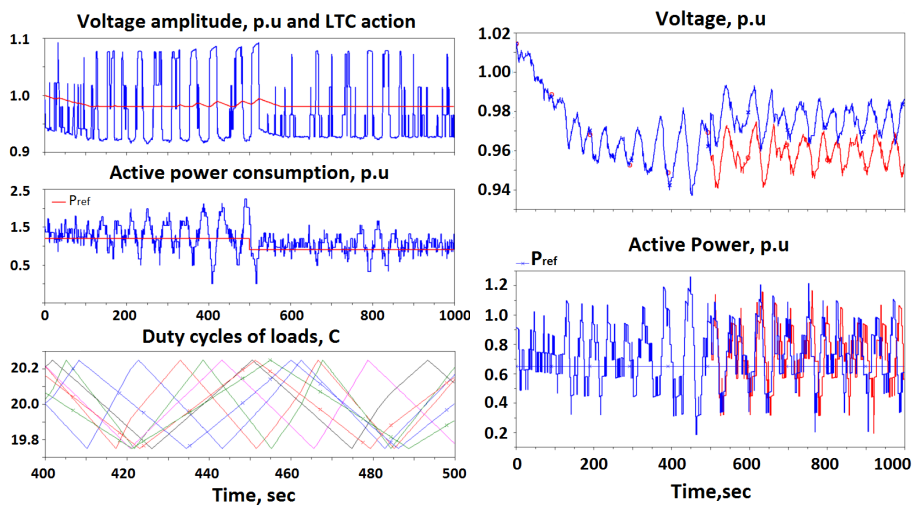

Fig. 6: Simulation results of the Fig. 7: Change in voltage and TCL loads $(n=22)$ for experiment active power consumption with $2 \mathrm{~b}$ with $23 \%$ load shedding: the capacitor bank activation (blue) voltage $V_{1}$ regulated by controller and without (red) capacitor bank (blue), the transform ratio $r$ (red) activation

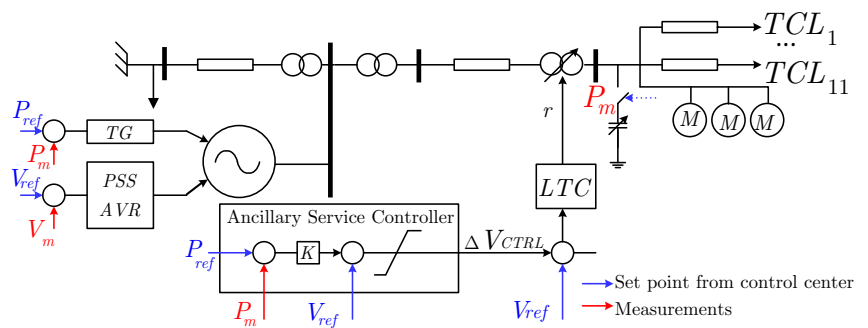

Fig. 8: Modified Greek power plant model [19] to include TCLs and capacitor bank switching

(Fig. 6). Without controlling of the loads state of switching on/off $(u(t)$ parameter in the TCL model (Section III-B), there is a very fast and simultaneous breach of the maximum temperature limit (with small difference due to heterogeneity). After that, an interruption in power consumption occurs for the portion of duty cycle that is not controlled due to voltage independence. One can also observe stretching of the power consumption cycle, and after, jamming with higher amplitude peaks. These are product of voltage changes over time. The voltage decrease causes a rise of the TCL duty cycle, while a voltage increase does the opposite.

When the controller attempts to follow the power consumption change in the intervals where homogeneous loads synchronize, the voltage change has a large variance around the mean value. In the case of high homogeneity in loads, this may create a problem. An obvious solution for such problem can be low pass filtering, which delays signal comparison and can decrease precision. This issue has to be taken into consideration when the control is designed to provide the ancillary service.

C. Case 3: Control in power consumption for a mixed load (motors and TCLs $(n=11)$ ) with capacitor bank activation

In this experiment of a Greek power plant model has been extended to include TCLs (Fig. 8) [19] and was used to model TCLs, inductive loads and to observe the influence of capacitor switching on measured outputs. The model consists of one generator, 3 motors, 11 TCL loads heterogeneous in thermal capacitance $C$, transformers and an infinite bus. The controller 
TABLE I: Numerical Experiment Results

\begin{tabular}{|c|c|c|c|c|c|}
\hline & \multirow{2}{*}{ Exp. Name } & \multirow[t]{2}{*}{ Signal } & No Fault & Small Perturbation (4.5\%) & Load Shedding (23\%) \\
\hline & & & Mean and Variance $(\mu, \sigma)$ & Before $(\mu, \sigma)$ After $(\mu, \sigma)$ & Before $(\mu, \sigma)$ After $(\mu, \sigma)$ \\
\hline \multirow[t]{2}{*}{$1 \mathrm{a}$} & No LTC, & $V, p . u$ & $(1.0277,0.0106)$ & $(1.0282,0.0106)(1.0295,0.0110)$ & $(1.0271,0.0106)(1.0352,0.0065)$ \\
\hline & no Control & $P, p . u$ & $(1.34,0.46)$ & $(1.3194,0.4689)(1.2585,0.4929)$ & $(1.3299,0.4324)(1.0150,0.3399)$ \\
\hline \multirow[t]{2}{*}{$1 \mathrm{~b}$} & LTC & $V, p . u$ & $(1.0089,0.0210)$ & $(1.0089,0.0210)(1.0113,0.0220)$ & $(1.0065,0.0215)(0.9905,0.0155)$ \\
\hline & no Control & $P, p . u$ & $(1.3244,0.4168)$ & $(1.3244,0.4168)(1.2713,0.4426)$ & $(1.3398,0.3854)(1.0223,0.3076)$ \\
\hline \multirow[t]{3}{*}{$2 \mathrm{a}$} & $\mathrm{LTC}+$ & $V, p . u$ & $(0.9318,0.0186)$ & $(0.9327,0.0185)(0.9444,0.0256)$ & $(0.9245,0.0113)(0.9337,0.0159)$ \\
\hline & Control & $P, p . u$ & $(1.1885,0.3914)$ & $(1.1896,0.3897)(1.1322,0.3552)$ & $(1.0387,0.2902)$ \\
\hline & $P_{\text {ref }}$ const & $P_{\text {ref }}, p . u$ & 1 & 1.2 & 1 \\
\hline \multirow[t]{3}{*}{$2 b$} & LTC + & $V, p . u$ & $(0.9836,0.0477)(0.9809,0.0209)$ & $(0.9749,0.0411)(1.0054,0.0338)$ & $(0.9302,0.0191)(0.9301,0.0098)$ \\
\hline & Control & $P, p . u$ & $(1.3237,0.3648)(1.2917,0.4016)$ & $(1.2956,0.3750)(1.2267,0.3565)$ & $(1.1947,0.3651)(0.9178,0.2612)$ \\
\hline & $P_{\text {ref }}$ step & $P_{\text {ref }}, p . u$ & 1.31 .25 & 1.31 .25 & 1.20 .9 \\
\hline
\end{tabular}

is installed on the LTC next to the load bus. In this case a first order filter function block is added in order to reduce power variation influence to voltage output (Figs. 8, 7). The capacitor bank activates when voltage hits the limit. In Fig. 7, it is possible to observe an improvement in voltage amplitude when comparing to the case without capacitor bank activation. This indicates that it is possible to enhance the controller's performance by coordinating it with capacitor bank switching.

\section{CONCLUSION AND FUTURE WORK}

The possibility to use efficiently all available tools to provide ancillary service is always of great interest and a key aim to electricity service providers. The work presented in this paper allows to introduce the use of voltage as a natural signal to control power consumption variations in a very favorable position. In the advent of fast power electronics as a solution for voltage control (new switches are going to substitute old LTCs), the current limitations in mechanical switching controls would not be a problem in the future.

Future work will be focused on developing a model predictive controller which can perform better than ordinary proportional control and which can enhance controller performance when considering discrete reactive power bank activation. In addition, there is a strong need to continue to work on model reduction of TCL ensembles to facilitate the TCL aggregation, modeling and simulation.

\section{APPENDIX A}

LTC descrete parameters are $U p_{\text {lim }}=0.01$, Down $n_{\text {lim }}=-0.01$, position $N o=32, r_{0}=1, Y_{\min }=0.97$ p.u., $Y_{\max }=1.03$ p.u., dela $y_{1}=30$ $\mathrm{s}$, delay $y_{2}=1 \mathrm{~s}$. LTC continuos (used in the controller): $V_{20}=1 p . u$., $t_{c}=179.2 \mathrm{~s}$. Line paremeters: $R=0.01 p u, X=0.1$ p.u., $G=0$, $B=0.001 / 2$ p.u.. Greek Power Plant parameters: Generator VI [20]: $S_{n}=100 \mathrm{MVA}, V_{n}=19 \mathrm{kV}, r_{a 0}=0.0028$ p.u., $x_{d 0}=2.08$ p.u., $x_{q 0}=2$ p.u., $x_{d 0}^{\prime}=0.305$ p.u., $x_{q 0}^{\prime}=0.49$ p.u., $x_{d 0}^{\prime \prime}=0.245$ p.u., $x_{q 0}^{\prime \prime}=0.245$ p.u., $t_{d 0}^{\prime}=6.8 \mathrm{~s}, t_{q 0}^{\prime}=0.62 \mathrm{~s}, t_{d 0}^{\prime \prime}=0.0402 \mathrm{~s}, t_{q 0}^{\prime \prime}=$ $0.077 \mathrm{~s}, t_{a a}=0 \mathrm{~s}, M_{0}=6.48 \mathrm{kWs} / \mathrm{kVA}$; Motor $1: \beta=1, v_{0}=1$ p.u., $P=0.032$ p.u., $Q=0.016$ p.u., $w_{m}=0.4954, V_{\text {bus }}=6.6 \mathrm{kV}, J=$ $0.499, I_{r}=323.3$; Motor2: $\beta=1, v_{0}=1$ p.u., $P=0.014$ p.u., $Q$ $=0.007$ p.u., $w_{m}=0.9914, V_{\text {bus }}=6.6 \mathrm{kV}, J=1.443, I_{r}=142.6$; Transformer: $X=0.0725$ p.u., $G=0, B=0, r=1, R=1 \mathrm{e}-010$ p.u.; Transformer: $X=0.15$ p.u., $G=0, B=0, r=1, R=1 \mathrm{e}-010$; PQ load: $P=8$ p.u., $Q=6$ p.u.; AVR TypeIII [20]: $v_{0}=1$ p.u., $K_{0}$ $=4.15, T_{2}=1, T_{1}=1, T_{e}=0.01 \mathrm{~s} ;$ TG TypeI [20]: $R=0.04, T_{s}$
$=1 \mathrm{~s}, T_{c}=0.3 \mathrm{~s}, T_{3}=0.04 \mathrm{~s}, T_{4}=5 \mathrm{~s}, T_{5}=4 \mathrm{~s}, p_{\max }=0.5$ p.u., $p_{\min }=0$

\section{REFERENCES}

[1] G. Heffner et al., "Loads providing ancillary services: Review of international experience," Lawrence Berkeley National Lab., May 2007.

[2] O. Ma et al., "Demand response for ancillary services," IEEE Transactions on Smart Grid, vol. 4, no. 4, pp. 1988-1995, Dec 2013.

[3] W. Zhang et al., "Aggregate model for heterogeneous thermostatically controlled loads with demand response," in IEEE Power and Energy Society General Meeting, July 2012.

[4] S. Meyn et al., "Ancillary service to the grid using intelligent deferrable loads," arXiv preprint arXiv:1402.4600, 2014.

[5] Nuclear Energy Agency, Technical and Economic Aspects of Load Following with Nuclear Power Plants, 2011.

[6] D. Callaway and I. Hiskens, "Achieving controllability of electric loads," Proceedings of the IEEE, vol. 99, no. 1, pp. 184-199, Jan 2011.

[7] K. P. Schneider, J. Fuller, F. Tuffner, and R. Singh, "Evaluation of conservation voltage reduction (cvr) on a national level," Pacific Northwest National Laboratory report, 2010.

[8] Electric Power Research Institute, "Measurement-based load modeling," Tech. Rep., December 2006.

[9] T. Van Cutsem and C. Vournas, Voltage stability of electric power systems. Springer, 1998.

[10] A. Borghetti et al., "On dynamic load models for voltage stability studies," IEEE Trans. on Power Systems, vol. 12, pp. 293 - 303, 1997.

[11] L. M. Hajagos and B. Danai, "Laboratory measurements and models of modern loads and their effect on voltage stability studies," IEEE Transactions on Power Systems, vol. 13, pp. 584 - 592, 1998.

[12] Y. V. Makarov, V. A. Maslennikov, and D. J. Hill, "Revealing loads having the biggest influence on power system small disturbance stability," IEEE Transactions on Power Systems, vol. 11, no. 4, pp. 2018 - 2023, 1996.

[13] M. M. C. de Almeida, "Study of load representation models in electrical distribution systems," Tech. Rep., 2004.

[14] D. Karlsson and D. J. Hill, "Modelling and identification of nonlinear dynamic loads in power systems," IEEE Transactions on Power Systems, vol. 9, no. 1, pp. 157-166, 1994.

[15] U.S. Department of Energy, "Application of Automated Controls for Voltage and Reactive Power Management - Initial Results," Electricity Delivery and Energy Reliability, Tech. Rep., December 2012.

[16] K. Turitsyn, P. Sulc, S. Backhaus, and M. Chertkov, "Options for control of reactive power by distributed photovoltaic generators," Proceedings of the IEEE, vol. 99, no. 6, pp. 1063-1073, 2011.

[17] J. Mathieu, S. Koch, and D. Callaway, "State estimation and control of electric loads to manage real-time energy imbalance," IEEE Transactions on Power Systems, vol. 28, no. 1, pp. 430-440, Feb 2013.

[18] G. Sybille, "Simpowersystems user's guide, version 4, published under sublicense from hydro-québec, and the mathworks, inc," 2004.

[19] T.Bogodorova, L.Vanfretti, and K.Turitsyn, "Bayesian parameter estimation of power system primary frequency controls under modeling uncertainties," in 17th IFAC Symposium on System Identification, October 2015.

[20] F. Milano, Power System Analysis Toolbox Documentation for PSAT Version 2.1.6, May 2010. 